\title{
Cluster mass estimation from lens magnification
}

\author{
Eelco van Kampen \\ Theoretical Astrophysics Center, \\ Juliane Maries Vej 30, DK-2100 Copenhagen Ø, Denmark \\ E-mail: eelco@tac.dk
}

\begin{abstract}
The mass of a cluster of galaxies can be estimated from its lens magnification, which can be determined from the variation in number counts of background galaxies. In order to derive the mass one needs to make assumptions for the lens shear, which is unknown from the variation in number counts alone. Furthermore, one needs to go beyond the weak lensing (linear) approximation as most of the observational data is concentrated in the central parts of clusters, where the lensing is strong. By studying the lensing properties of a complete catalogue of galaxy cluster models, one can find reasonable approximations about the lens shear as a function of the lens convergence. We show that using these approximations one can fairly well reconstruct the surface mass distribution from the magnification alone.
\end{abstract}

\section{Motivation}

A rich cluster of galaxies acts as a gravitational lens on the galaxy distribution beyond it. This simple fact can be used to derive a great deal about both the lensing cluster as well as the background galaxy population. Here we discuss how to best exploit the variation in galaxy number counts caused by the lensing cluster, which enables one to derive the lens magnification 1 . Recently, is has been shown that a depletion in number counts can clearly be observed for the clusters $\mathrm{Cl} 0024+1654$ 2 and A16893.

However, in order to obtain a mass for the lens, or even a mass distribution, one needs the lens shear as well, because the lens magnification $\mu$, shear $\gamma$ and convergence $\kappa$ (the dimensionless surface mass density) are related as $\mu^{-1}=(1-\kappa)^{2}-\gamma^{2}$.

There are ways for obtaining the shear from observations 1 , but one would like to obtain a mass estimate that is independent of other methods. This means we need to find assumptions for the shear, either as a function of surface mass density, or as a function of the lens magnification. We use a sample of numerical galaxy cluster models $\mathrm{E}$ to find heuristic relations between $\gamma$ and $\kappa$ (or $\mu$ ), some of which will have an underlying assumption about the physical state of the lens, like isotropy. 


\section{Estimating the lens convergence from lens magnification}

We wish to find a local relation for $\gamma$ vs. $\kappa$ and/or $\mu$. The simplest one is the weak lensing approximation, $\kappa_{\text {lin }}=(1-\mu) / 2$, which is valid only in the linear, small- $\kappa$ regime 1 , i.e. in the outskirts of clusters. However, most observational data is available for the central parts of clusters, where this approximation is invalid, and we need to go beyond the weak lensing approximation to realistically estimate the cluster surface mass density.

There are only two local relations between $\gamma$ and $\kappa$ that result in a single caustic solution of the magnification equation which is easily invertiblel: $\gamma=0$, corresponding to a sheet of matter, and $\gamma=\kappa$, for an isotropic lens. In the shearless case we have the estimate $\kappa_{0}=1-\mathcal{P}|\mu|^{-1 / 2}$, while for the isotropic case the estimator becomes $\kappa_{1}=\left(1-\mathcal{P}|\mu|^{-1}\right) / 2$, where the $\mathcal{P}$ is the image parity, i.e. the sign of $\mu$. Note that one can only measure $|\mu|$, and therefore $\mathcal{P}$ has to be assigned by hand.

In practice, substructure and asphericity of the cluster will induce extra shear, especially in the surrounding low- $\kappa$ neighbourhood, where substructure is relatively more dominant and filaments make the cluster most aspherical. An approximation that tries to take these cluster lens features into account, while still giving an invertible $\mu(\kappa)$ relation, is $\gamma=\sqrt{\left(c+c^{-1}-2\right) \kappa}$. This results in the amplification relation $\mu^{-1}=(\kappa-c)\left(\kappa-c^{-1}\right)$, with caustics at $\kappa=c$ and $\kappa=c^{-1}$. The solution for $\kappa$ is then 6

$$
\kappa_{c}=\frac{c+c^{-1}}{2}+\mathcal{S}\left[\left(\frac{c+c^{-1}}{2}\right)^{2}-\mathcal{P}|\mu|^{-1}-1\right]^{1 / 2},
$$

where we have introduced a second parity $\mathcal{S}$ which is the sign of $\kappa-\left(c+c^{-1}\right) / 2$. Note that the $\gamma=0$ approximation is recovered by setting $c=1$.

\section{Testing the convergence estimators on cluster models}

Although the feasibility of actually obtaining $\mu$ from real data is an interesting topic for discussion, the issue here is how to proceed from the measured magnification to other properties of the lensing cluster, notably its mass distribution. We therefore assume here that one can reliably measure the lens magnification, and investigate how well the approximations allow us to reconstruct the lens convergence from just this magnification map. This should show us the best possible result each approximation can provide us, and reveals systematics.

Using the thin lens approximation 8 , we produce maps of the lens convergence, shear and magnification for the cluster models. We then use the magnification only (with full knowledge of its parity, though), to reconstruct 

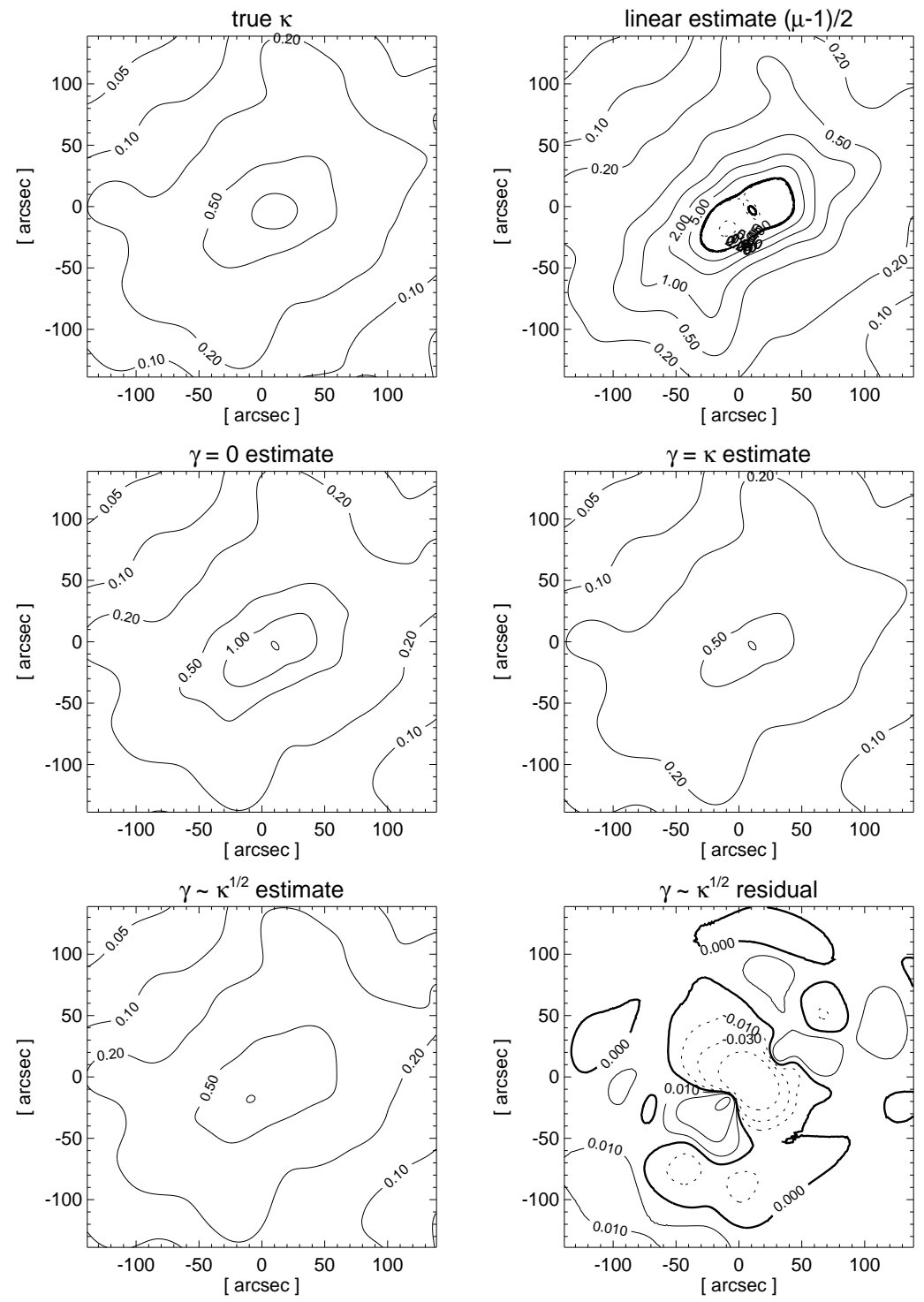

Figure 1: True versus estimated $\kappa$-maps for a numerical model of a rich cluster. The top-left panel shows the true $\kappa$, the next four panels show the estimates for the linear, $\gamma=0, \gamma=\kappa$, and $\gamma \propto \kappa^{1 / 2}$ approximations, while the bottom-right panel shows the difference of the latter estimate compared to the true distribution (i.e. top-left panel minus bottom-left panel). 
convergence maps using the various assumptions about the shear, and compare these to the true convergence. This is shown in Fig. 1 for the most massive model cluster from the catalogue, as this one has the largest range of possible values for the convergence $\kappa$.

The linear estimator performs very poorly, as it just follows the magnification, including the caustics. It is only doing well for small $\kappa$, as expected 1 . The $\gamma=0$ assumption produces an overestimate for the convergence for all regions of the cluster. The $\kappa=\gamma$ estimator underestimates the mass in the central regions of the cluster, and (slightly) overestimates for $\kappa<0.2$. The $\gamma \propto \sqrt{\kappa}$ estimator clearly performs best.

\section{Discussion}

The fact that these estimators can reproduce the surface mass density reasonable well for model clusters does not guarantee that they work on observational data. The best performing estimator has the clear disadvantage that two parities need to be set. More generally, one has to deal with many intrinsic sources of error associated with observed magnification maps. Because the number of background galaxies that can be used to construct a magnification map will be finite, there will be shot noise. Therefore one needs to smooth the distribution of number counts, or average over annular bins. Furthermore, the backgrond galaxies are clustered and have different redshifts, which produces extra uncertainty. However, these problems can be dealt with to some extent 1 , 1 , and the magnification method, employing our strong lensing estimators, seems a feasible way to directly measure the dark matter distribution of galaxy clusters.

\section{References}

1. T.J. Broadhurst, A.N. Taylor, J.A. Peacock, 1995, ApJ, 438, 49

2. B. Fort, Y. Mellier, M. Dantel-Fort, 1997, A\&A, 321, 353

3. A.N. Taylor, S. Dye, T.J. Broadhurst, N. Benítez, E. van Kampen, 1997, submitted to ApJ

4. N. Kaiser, G. Squires, 1993, ApJ, 404, 441

5. E. van Kampen, P. Katgert, 1997, MNRAS, 289, 327

6. E. van Kampen, 1997, submitted to MNRAS

7. M. Bartelmann, M. Steinmetz, A. Weiss, 1995, A\&A, 297, 1

8. M. Bartelmann, A. Weiss, 1994, A\&A, 287, 1

9. A.N. Taylor, S. Dye, 1997, submitted to MNRAS 